\title{
ARTICLE Nucleus accumbens volume as a predictor of anxiety symptom improvement following CBT and SSRI treatment in two independent samples
}

\author{
Katie L. Burkhouse ${ }^{1,2}$, Jagan Jimmy ${ }^{1}$, Nicholas Defelice ${ }^{1}$, Heide Klumpp ${ }^{1,2}$, Olusola Ajilore ID $^{1}$, Bobby Hosseini ${ }^{1}$, Kate D. Fitzgerald ${ }^{3}$,
} Christopher S. Monk ${ }^{3,4}$ and K. Luan Phan ${ }^{1,5}$

Structural variations of neural regions implicated in fear responses have been well documented in the pathophysiology of anxiety and may play an important role in treatment response. We examined whether gray matter volume of three neural regions supporting fear and avoidance responses [bilateral amygdala, nucleus accumbens (NAcc), and ventromedial prefrontal cortex (PFC)] predicted cognitive-behavioral therapy (CBT) and selective serotonin reuptake inhibitor (SSRI) treatment outcome in two independent samples of patients with anxiety disorders. Study 1 consisted of 81 adults with anxiety disorders and Study 2 included 55 children and adolescents with anxiety disorders. In both studies, patients completed baseline structural MRI scans and received either CBT or SSRI treatment. Clinician-rated interviews of anxiety symptoms were assessed at baseline and posttreatment. Among the adult sample, greater pre-treatment bilateral NAcc volume was associated with a greater reduction in clinician-rated anxiety symptoms pre-to-post CBT and SSRI treatment. Greater left NAcc volume also predicted greater decreases in clinician-rated anxiety symptoms pre-to-post CBT and SSRI treatment among youth with current anxiety. Across studies, results were similar across treatments, and findings were maintained when adjusting for patient's age, sex, and total intracranial brain volume. We found no evidence for baseline amygdala or ventromedial PFC volume serving as treatment predictors across the two samples. Together, these findings provide promising support for the role of NAcc volume as an objective marker of anxiety treatment improvement that spans across development. Future studies should clarify the specific mechanisms through which NAcc volume exerts its therapeutic effects.

Neuropsychopharmacology (2020) 45:561-569; https://doi.org/10.1038/s41386-019-0575-5

\section{INTRODUCTION}

Anxiety disorders are among the most prevalent internalizing psychopathologies and are associated with significant economic burden worldwide [1, 2]. Current first-line treatment for anxiety disorders consists of cognitive-behavioral therapy (CBT) and/or selective serotonin reuptake inhibitor (SSRI) treatment $[3,4]$. However, response rates to these treatments range from 38 to $87 \%$ across anxiety diagnoses $[5,6]$ and often worsen in the context of comorbid psychopathology [7]. Importantly, a diagnosis of anxiety rarely occurs in isolation, with substantial homotypic (secondary anxiety disorder) and heterotypic (secondary other psychiatric disorder) comorbidity rates documented among youth [8] and adult [9] populations. Given this, identifying predictors of treatment response for patients with heterogeneous anxiety disorders is essential to inform decision-making processes and improve patient outcomes to reduce the burden on both the individual and society.

Structural variations of neural regions implicated in fear and avoidance responses have been well documented in the pathophysiology of anxiety disorders [10] and may play an important role in treatment response. Utilizing fear conditioning and avoidance models, studies have identified several neural regions involved in fear circuitry and behaviors, including the ventromedial prefrontal cortex (PFC), nucleus accumbens (NAcc), and limbic areas (e.g., amygdala, hippocampus, and insula) [11-13]. The amygdala plays an important role in the perception, learning, and early extinction of threat [14], whereas the ventromedial PFC is thought to play a significant part in the retention of fear extinction [15] as well as the ability to use adaptive regulation strategies to attenuate aversive responses to threat [16]. Researchers have also shown that the NAcc plays a role in the etiology and maintenance of aberrant fear avoidance behaviors among individuals with anxiety [11, 17], and the degree of NAcc activation modulates positive associations between anxiety levels and responses to fear-evoking stimuli [18].

Across several studies, anxiety disorders are characterized by structural gray matter differences in neural regions supporting fear responses. For instance, generalized anxiety disorder (GAD), panic disorder, post-traumatic stress disorder (PTSD), social anxiety disorder (SAD), and specific phobia are defined by decreased amygdala volume across youth and adult samples [19-23]. Decreases in amygdala gray matter volume have also been

\footnotetext{
${ }^{1}$ Department of Psychiatry, University of Illinois at Chicago, Chicago, IL, USA; ${ }^{2}$ Department of Psychology, University of Illinois at Chicago, Chicago, IL, USA; ${ }^{3}$ Department of

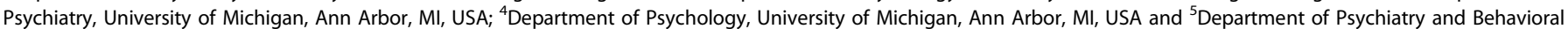
Health, The Ohio State University, Columbus, $\mathrm{OH}$, USA Correspondence: Katie L. Burkhouse (kburkho@uic.edu)
}

Received: 30 August 2019 Revised: 1 November 2019 Accepted: 11 November 2019 Published online: 22 November 2019 
562

documented among twin pairs concordant for anxiety and depression [24], suggesting that amygdala reduction is related to genetic risk for internalizing disorders. It has been suggested that persistent amygdala hyperactivation, often observed among patients with anxiety, may lead to glutamatergic excitotoxicity, which subsequently reduces amygdala volumes [25]. On the other hand, studies have also identified larger amygdala volumes among patients with GAD [26, 27]; however, these studies are limited by small sample sizes. Other research has shown that SAD is characterized by greater ventromedial PFC volume, which may contribute to progressive modulation of excessive limbic activity in response to threat-relevant material [28]. Finally, separate research suggests that greater bilateral NAcc volume is observed among adults with GAD [29] and associated with higher levels of trait anxiety [30]. These documented alterations in NAcc volume among individuals with anxiety may be a driving mechanism underlying motivation for avoidance behaviors in response to fear-relevant stimuli [31].

Despite recent evidence that neural biomarkers, vs. demographic and clinical data, improve accuracy in predicting treatment response $[32,33]$, few studies to date have explored whether regional brain volume of structures supporting fear responses may serve as a predictor of treatment response for patients with anxiety. In one study, larger rostral anterior cingulate cortex volume was associated with better CBT response for patients with PTSD [34]. Other studies have shown that CBT response is predicted by greater hippocampal volume for patients with PTSD [35] and panic disorder [36]. These prior studies, however, did not explore the predictive role of other brain regions supporting fear and avoidance responses that have shown to be disrupted in anxiety disorders (i.e., bilateral amygdala, ventromedial PFC, and NACC) among patients with heterogeneous anxiety disorders. Indeed, first-line treatments for anxiety, such as CBT and SSRI treatment, work to target fear responses $[37,38]$ and there is increasing evidence that these brain regions supporting fear responses are altered by effective anxiety treatment. For example, following treatment with CBT or SSRIs, amygdala activation to threat-relevant material is decreased $[39,40]$, whereas ventromedial PFC activation is enhanced among adults with anxiety [41]. CBT also enhances amygdala-ventromedial PFC functional connectivity among adults with social anxiety [42]. Regarding treatment prediction, there is evidence that pre-treatment amygdala activation in response to threat-relevant stimuli predicts CBT and SSRI treatment response for anxiety across child and adult populations [40,43-45]; however, the direction of activation is mixed across studies. Other research has found that greater baseline activation in prefrontal regions involved in appraising and regulating responses to threatening stimuli is associated with better CBT and SSRI treatment response in studies of anxious youth and adults $[46,47]$.

Together, these previous studies provide evidence that neural regions supporting fear processing are well implicated in anxiety disorders across development and may be useful predictors for determining whether individuals with anxiety will respond to treatment. Notably, most previous treatment prediction studies with anxious samples have focused on functional, vs. structural, magnetic resonance imaging (MRI) predictors. A strength of examining structural MRI predictors is the ability to easily compare results across studies, whereas differences across fear and avoidance paradigms may obscure findings across functional MRI studies. In addition, structural MRI parameters have demonstrated high retest reliability [48], whereas meta-analytic studies show poor reliability for taskrelated fMRI [49] making $\mathrm{fMRI}$ measures less suitable as clinical biomarkers or treatment predictors. Thus, the primary aim of the current study was to examine whether individual differences in pre-treatment gray matter volume of regions supporting fear and avoidance responses predict anxiety symptom change following CBT and SSRI treatment among adults with heterogeneous anxiety disorders. We chose to utilize an a priori region of interest (ROI) approach focusing on neural regions supporting fear and avoidance responses that have shown to be disrupted in anxiety disorders (i.e., bilateral amygdala, ventromedial PFC, and NACC).

The current study also sought to test these structural MRI predictors in a separate independent sample of treatment-seeking children and adolescents with anxiety disorders. The highest risk period for developing an anxiety disorder is during the child and adolescent period [50]; thus, identifying biological predictors of intervention response for this developmental population is critical. Notably, no studies to date exploring neural predictors of treatment response for anxiety disorders have included a separate independent sample to determine if effects generalize to other populations. Reproducibility of findings is also important for the neuroscience field, as research suggests that structural and functional MRI studies tend to have small sample sizes with low statistical power and replication rates [51, 52]. The two independent studies included were comparable in study design and patient characteristics such that they included patients (adults or youth) with heterogeneous anxiety disorders (similar in composition of diagnoses) who completed a baseline structural MRI scan prior to receiving CBT or SSRI treatment.

\section{METHODS}

Study 1 -adults

Participants and procedure. Study 1 involved a treatment-seeking community sample of adults $(n=81)$ between the ages of 18 and 50 with a current anxiety disorder. To be included as a patient, participants were required to meet the following criteria: a current full-threshold DSM-5 anxiety disorder, a Global Assessment of Functioning score of $\leq 60$, and report a total score of $\geq 23$ on the Depression, Anxiety, and Stress Scale (DASS-21) [53]. Exclusionary criteria for the patient group included an inability to provide consent and read and write in English; a major active medical or neurological problem; a history of mania, psychosis, an intellectual disability or pervasive developmental disorder; current substance dependence; any contraindication to receiving SSRIs; being currently enrolled in psychiatric treatment; a history of traumatic brain injury; and being pregnant. This study was approved by the UIC Institutional Review Board and informed consent was obtained from all participants. The study was a parallel group randomized control trial with 1:1 allocation ratio registered on ClinicalTrials.gov (Identifier: NCT01903447; see Supplementary Fig. 1 for Consort Diagram). All participants were monetarily compensated for their time.

Assessment of psychopathology. Psychiatric diagnoses were assessed via the SCID-5 [54] by a trained masters-level assessor, PhD-level psychologist, or psychiatrist. The breakdown of current anxiety diagnoses was $75.3 \%(n=61) \mathrm{GAD}, 65.4 \%(n=53) \mathrm{SAD}$, $28.4 \%(n=23)$ panic disorder, $19.8 \%(n=16)$ specific phobia, $17.3 \%(n=14)$ PTSD, and $8.6 \%(n=7)$ agoraphobia. Patients also had other current comorbid psychiatric disorders including major depressive disorder $(n=38,46.9 \%)$ and persistent depressive disorder $(n=13,16 \%)$. Eighty-nine percent of patients $(n=72)$ had comorbid psychopathologies. Of the nine with a single anxiety disorder, 66.7\% $(n=6)$ had GAD, $22.2 \%(n=2)$ had SAD, and $11.1 \%$ had panic disorder $(n=1)$.

At pretreatment and posttreatment, trained clinical research assessors blinded to patient treatment randomization administered participants the Hamilton Anxiety Rating Scale (HAM-A) [55], a 14-item interview-based measure of broad anxiety and somatic symptoms, and the Hamilton Depression Rating Scale (HAM-D) [56], a 17-item interview-based measure of broad depressive and somatic symptoms. 
Treatment procedures. Participants were randomized to either 12 weeks of CBT $(n=41)$ or SSRI $(n=40)$ treatment. For participants randomized to SSRIs (sertraline, fluoxetine, paroxetine, escitalopram, or citalopram), the dosing schedule was flexible depending on tolerability and aimed to reach target dose by week 8 . Patients receiving SSRIs attended medication management sessions that lasted approximately 20-30 min with their study psychiatrist at $0,2,4,8$, and 12 weeks. For participants receiving $C B T$, treatment was delivered through 12 , once-weekly 60-min sessions led by a PhD-level clinical psychologist under the supervision of a licensed clinical psychologist with expertize in CBT. Evidence-based manuals were used based on the patient's principal diagnosis and predominant symptoms [57-59]. As per the manualized protocol, sessions began with psychoeducation and cognitive restructuring and then expanded to include strategies such as exposures and relapse prevention.

Structural MRI acquisition. The structural MRI scans were obtained on a $3 \mathrm{~T}$ GE Discovery System (General Electric Healthcare, Waukesha, WI) with an 8-channel head coil. After positioning the subjects in a supine position in the scanner, structural scans were obtained with a 3D BRAVO pulse sequence with the following parameters: flip angle $13^{\circ}$, inversion time $450 \mathrm{~ms}$, field of view $22 \times 22 \mathrm{~cm}$, matrix size $256 \times 256$, slice thickness $1 \mathrm{~mm}^{3}, 182$ axial slices of the whole brain.

\section{Study 2-youth}

Participants and procedure. Participants in the study were youth $(n=55)$ between the ages of 7 and 19 enrolled in a pediatric anxiety treatment study at the University of Michigan (UM) $(n=$ 29) and University of Illinois at Chicago (UIC) $(n=26)$. To be included in the study, participants must have met criteria for a current diagnosis of GAD or SAD. Exclusion criteria included history of bipolar disorder, schizophrenia, intellectual disability, pervasive development disorders, current substance use disorders, severe depression, or suicidal ideation. Participants were not taking psychotropic medications or in psychotherapy for at least 4 weeks prior to the initial assessment. Procedures were approved by the Institutional Review Boards at both UIC and UM and informed consent and assent were obtained from all participants.

Assessment of psychopathology. The Kiddie Schedule for Affective Disorders and Schizophrenia for School-Age Children [60] diagnostic interview was used to assess for diagnoses by Master's or Doctoral level clinicians. The breakdown of current diagnoses was $70.9 \%$ GAD $(n=39), 56.4 \%$ SAD $(n=31), 16.4 \%$ specific phobia $(n=9), 14.5 \%$ separation anxiety disorder, $10.9 \%$ panic disorder $(n=6), 5.5 \%$ obsessive compulsive disorder $(n=3)$, and $1.8 \%$ PTSD $(n=1)$. Participants with secondary depressive disorders were included in the study $(5.5 \%$ with current major depression, $n=3)$. Totally, $58 \%$ of patients $(n=32)$ had comorbid psychopathologies. Of the twenty-three with a single anxiety disorder, 60.9\% $(n=14)$ had GAD, and 39.1\% $(n=9)$ had SAD.

At pre- and post-treatment, trained clinical research assessors blinded to patient treatment administered participants the Pediatric Anxiety Rating Scale (PARS) [61]. Masters-level clinicians rated overall anxiety severity on a six-point scale across seven dimensions [number of symptoms (GAD, SAD, and separation anxiety), frequency, overall severity, physical symptom severity, avoidance, and interference in and outside of the home], with these dimensions then combined to form a total PARS score. Youth also completed the Children's Depression Inventory (CDI) [62], a 27-item measure of youth's depressive symptoms.

Treatment procedures. At UM, participants were offered and selfselected treatment with SSRI or CBT. At UIC, participants were initially randomly assigned to receive either an SSRI or CBT, but could opt to switch from SSRI to CBT due to intolerable side effects. SSRI treatment consisted of 12 weeks of sertraline prescribed by a child psychiatrist during medication management sessions, beginning with a dose of 12.5 or $25 \mathrm{mg} /$ day and in a flexible-dosing design increasing on subsequent visits up to $200 \mathrm{mg} /$ day based on tolerability and treatment response. CBT was delivered through weekly 60-min sessions (up to a maximum of 18 sessions) by a Master's or Doctoral level therapist under the supervision of a licensed clinical psychologist with expertize in CBT. Treatment followed an established manualized CBT intervention for pediatric anxiety [63, 64].

Structural MRI acquisition. At UIC, the acquisition parameters were identical to those described above in the adult study. At UM, a 3.0 T GE Signa Scanner (General Electric; Milwaukee, Wisconsin, USA) with a GE quad head coil was used to acquire high resolution, T1-weighted volumetric anatomical scans (3D spoiledgradient echo sequence, $9 \mathrm{~ms}$ repetition time, $1.8 \mathrm{~ms}$ echo time, $500 \mathrm{~ms}$ inversion time, $15^{\circ}$ flip angle, $256 \times 256$ matrix, $256 \mathrm{~mm}$ field of view; 124 slices, $1.2 \mathrm{~mm}$ slice thickness).

Structural MRI preprocessing. Across both studies, the brain structural measurements were obtained using FreeSurfer Image analysis suite version 6.0 (http://freesurfer.net/fswiki/ FreeSurferWiki). Automated procedure for cortical reconstruction and volumetric segmentation has been previously documented in detail $[65,66]$. In short, structural images analysis involved motion correction, removal of non-brain tissue, transformation to Talairach space, segmentation of white matter and gray matter structures, intensity normalization, tessellation of gray-white matter boundary, topology correction, and surface deformation following intensity gradients for optimal placement of gray-white matter boundary and cerebrospinal fluid and gray matter boundary. The reconstructed cortical surface was automatically parcellated to cortical units with respect to gyral and sulcal structure based on probabilistic information from previously labeled atlases $[67,68]$ following which, the surface area, thickness, and volume of each unit were computed $[69,70]$. The subcortical structures were segmented and labeled using the probabilistic atlas provided by freesurfer and the subject-specific measured values [66]. This automated segmentation and labeling procedure has been shown to be of equal accuracy to manual tracing methods and relatively insensitive to changes in acquisition parameters [68]. The reconstruction and segmentation were visually inspected for any major estimation errors. The volume of the segmented structures (bilateral amygdala, ventromedial PFC, and NACC) were then estimated and extracted.

Data analysis plan. For both studies, a series of planned withinsubjects and between-subjects analysis of variance (ANOVA) tests were conducted to verify that treatment was successful in reducing anxiety symptoms. We also conducted a series of partial correlations to examine whether age or anxiety symptoms were correlated with brain volumes at baseline, controlling for total intracranial volume (ICV in $\mathrm{mm}^{3}$, derived from automated brain segmentation). A between-subjects ANOVA test was also conducted to explore gender differences in brain volumes at baseline, controlling for total ICV. Site (UIC or UM) was also entered as a covariate in all analyses for the youth study.

To examine whether brain volume at T1 predicted change in symptoms during treatment with CBT and/or SSRIs within patients, hierarchical linear regression analyses were conducted. For all models, brain volume ( $\mathrm{L}$ or $\mathrm{R}$ amygdala/ventromedial PFC/NAcc) at $\mathrm{T} 1$ (centered), ICV, anxiety symptoms at T1, and treatment arm (CBT, SSRI) were entered in Step 1, and the two-way interaction between T1 brain volume and treatment arm was entered in Step 2. Site (UIC or UM) also served as a covariate in the youth study models. Percent change in anxiety symptoms [([pretreatment-posttreatment symptoms] $\div$ pretreatment symptoms) $\times 100$ ] served as the dependent variable. 


\section{RESULTS}

Study 1-adults

Descriptive and clinical characteristics. Table 1 provides demographic and clinical characteristics of the adult sample separated by treatment group. Anxiety (HAMA: $F(1,79)=206.86, p<0.001$; $\eta_{p}{ }^{2}=0.72$ ) symptoms decreased pretreatment to posttreatment. Neither baseline anxiety symptoms nor the extent of reduction in anxiety symptoms pre-to-post treatment differed based on treatment modality $\left(p_{s}>0.07\right)$.

Brain volume and age, gender, and baseline anxiety correlations. Partial correlations revealed that age was negatively associated with R-NAcC $(r=-0.24, p=0.03)$ and R-ventromedial PFC $(r=-0.26, p=0.02)$ volume. Results revealed no other significant associations between age and brain volumes (lowest $p=0.08$ ). Results revealed that males, relative to females, exhibited greater bilateral amygdala volumes [left: $F(1,80)=11.06, p<0.01$, right: $F$ $(1,80)=10.54, p<0.01]$. No other analyses reached significance (lowest $p=0.24$ ). Gender and age were entered as covariates in all subsequent brain volume analyses.

Partial correlations revealed that reduced left amygdala volume was associated with greater anxiety severity at baseline $(r=-0.27$, $p=0.02$ ). Results revealed no other significant associations between age and brain volumes (lowest $p=0.10$ ). Baseline brain volumes did not differ based on treatment modality $\left(p_{s}>0.15\right)$.

$\mathrm{ROI}$ volume and treatment prediction. As shown in Table 2, results revealed significant main effects of T1 bilateral NAcc volume for change in anxiety symptoms. Specifically, greater T1 left NAcc volume was associated with a greater reduction in anxiety symptoms pre-to-post treatment (Fig. 1a), accounting for approximately $14 \%\left(R^{2}=0.14\right)$ of the variance in treatment response (vs. $6 \%$ without brain volume in the model). Similarly, greater T1 right NAcc volume also predicted greater anxiety improvement (Fig. 1b), accounting for approximately $16 \%\left(R^{2}=0.16\right)$ of the variance in treatment response. Results also revealed a significant T1 left amygdala $\times$ Arm (CBT or SSRI) interaction for change in anxiety symptoms, accounting for approximately $14 \%\left(R^{2}=0.14\right)$ of the variance in treatment response. However, follow-up analyses indicated that $\mathrm{T} 1$ left amygdala volume was not associated with anxiety symptom change among patients assigned to CBT $(\beta=-0.26, t=-1.28, \quad p=0.21)$ or SSRI treatment $(\beta=0.27$, $t=1.34, p=0.19$ ). As shown in Table 2 , no other analyses reached significance.

Post hoc analyses were then conducted to evaluate the predictive ability of bilateral NAcc volume. Receiving operating characteristic (ROC) curve analyses were conducted to predict responder status. Of the 81 patients, 61 (75\%) were classified as responders (defined as $>50 \%$ reduction in HAM-A symptoms). Findings revealed responder status was predicted by greater baseline left (Area $=0.66, p=0.04$; Supplemental Fig. 2a) and right (Area $=0.70, p<0.01$; Supplemental Fig. 2b) NAcc volume (larger result indicates more positive test).

\section{Study 2-Youth}

Descriptive and clinical characteristics. Table 1 provides patient characteristics of the youth sample separated by treatment group. Anxiety (PARS: $F(1,53)=190.27, p<0.001 ; \eta_{p}{ }^{2}=0.78$ ) symptoms decreased pretreatment to posttreatment. Neither baseline anxiety symptoms nor the extent of reduction in anxiety symptoms pre-to-post treatment differed based on treatment modality $\left(p_{s}>0.64\right)$.

ROI volume and age, gender, and baseline anxiety correlations. Partial correlations revealed that age was negatively associated with bilateral NAcc volume (left: $r=-0.41, p<0.01$, right: $r=-0.36, p<0.01)$. Results revealed no other significant age associations (lowest $p=0.29$ ). A series of between-subjects
Table 1. Demographic and clinical characteristics separated by treatment arm.

\begin{tabular}{|c|c|c|c|c|}
\hline Study 1 (Adult) & $\begin{array}{l}\mathrm{CBT}(N=41) \\
\mathrm{M}(\mathrm{SD})\end{array}$ & $\begin{array}{l}\text { SSRI }(N=40) \\
\mathrm{M}(\mathrm{SD})\end{array}$ & $t$ Value & $p$ Value \\
\hline Age & $27.80(8.98)$ & $25.70(7.71)$ & 1.13 & 0.26 \\
\hline T1 HAM-A symptoms & $16.15(5.19)$ & $18.73(6.93)$ & -1.85 & 0.08 \\
\hline T2 HAM-A symptoms & $5.85(4.58)$ & $7.13(6.02)$ & -1.07 & 0.29 \\
\hline TI HAM-D symptoms & $11.76(3.66)$ & $12.83(4.72)$ & -1.14 & 0.26 \\
\hline T2 HAM-D symptoms & $\begin{array}{c}4.68(3.71) \\
\qquad(\%)\end{array}$ & $\begin{array}{c}5.68(4.56) \\
\quad N(\%)\end{array}$ & $\begin{array}{l}-1.08 \\
x^{2}\end{array}$ & 0.29 \\
\hline Female & 29 (70.7\%) & $29(72.5 \%)$ & 0.03 & 0.53 \\
\hline Caucasian & 27 (65.9\%) & $25(62.5 \%)$ & 0.10 & 0.47 \\
\hline African American & 8 (19.5\%) & $6(15.0 \%)$ & 0.29 & 0.41 \\
\hline Asian & $4(9.8 \%)$ & $4(10.0 \%)$ & 0.00 & 0.63 \\
\hline American Indian & $0(0.0 \%)$ & $1(2.5 \%)$ & 1.04 & 0.49 \\
\hline Biracial/other & $2(4.9 \%)$ & $4(10.0 \%)$ & 0.77 & 0.33 \\
\hline Hispanic ethnicity & $7(1 \%)$ & $9(22.5 \%)$ & 0.38 & 0.37 \\
\hline Study 2 (Youth) & $\begin{array}{c}\text { CBT }(\boldsymbol{N}=28) \\
\mathbf{M}(\mathbf{S D})\end{array}$ & $\begin{array}{c}\text { SSRI }(\mathbf{N}=27) \\
\mathbf{M}(\mathbf{S D})\end{array}$ & $t$ Value & \\
\hline Age & $12.67(3.75)$ & $14.81(2.83)$ & -2.37 & 0.02 \\
\hline T1 PARS symptoms & $23.46(4.29)$ & $23.63(4.67)$ & -0.14 & 0.89 \\
\hline T2 PARS symptoms & $10.04(6.83)$ & $10.59(6.28)$ & -0.32 & 0.75 \\
\hline T1 CDI symptoms & $11.82(6.84)$ & $15.56(8.01)$ & -1.86 & 0.08 \\
\hline T2 CDI symptoms & $\begin{array}{c}5.21(5.45) \\
\quad N(\%)\end{array}$ & $\begin{array}{c}6.30(7.09) \\
\boldsymbol{N}(\%)\end{array}$ & $\begin{array}{l}-0.64 \\
x^{2}\end{array}$ & 0.53 \\
\hline Female & 15 (53.5\%) & $17(62.9 \%)$ & 0.50 & 0.33 \\
\hline Caucasian & 15 (53.5\%) & 19 (70.3\%) & 1.64 & 0.16 \\
\hline African American & $2(7.1 \%)$ & $1(3.7 \%)$ & 0.32 & 0.51 \\
\hline Asian & $0(0.0 \%)$ & $2(7.4 \%)$ & 2.15 & 0.24 \\
\hline Native Hawaiian & $0(0.0 \%)$ & $1(3.7 \%)$ & 1.06 & 0.49 \\
\hline Biracial/other & 11 (39.3\%) & $4(14.8 \%)$ & 4.15 & 0.07 \\
\hline Hispanic ethnicity & $8(28.6 \%)$ & $4(14.8 \%)$ & 2.41 & 0.30 \\
\hline
\end{tabular}

$C B T$ cognitive-behavioral therapy, SSRI selective serotonin reuptake inhibitor, HAM-A Hamilton anxiety rating scale, HAM-D Hamilton depression rating scale, PARS pediatric anxiety rating scale, $C D /$ children's depression inventory, $T 1$ pretreatment, $T 2$ posttreatment

ANOVA tests indicated no significant differences between males and females in brain volumes (lowest $p=0.09$ ). Age and gender were entered ascovariates in all subsequent brain volume analyses. No significant associations were observed between baseline anxiety severity and ROI volumes (lowest $p=0.12$ ).

ROI volume and treatment prediction. As shown in Table 3, results revealed a significant main effect of $\mathrm{T} 1$ left NAcc volume for change in anxiety symptoms. Specifically, greater T1 left NAcc volume was associated with a greater reduction in anxiety symptoms among youth pre-to-post treatment (Fig. 2), accounting for approximately $19 \%\left(R^{2}=0.19\right)$ of the variance in treatment response (vs. $8 \%$ without brain volume in the model). As shown in Table 3, no other analyses reached significance. Post hoc analyses were also conducted to evaluate the predictive ability of NAcc volume utilizing ROC curve analysis. Of the 55 youth, 33 (60\%) were classified as responders (defined as $>50 \%$ reduction in PARS symptoms). Findings revealed responder status was predicted by greater baseline left NAcc volume (Area $=0.66, p=0.04$; Supplemental Fig. 3). 


\begin{tabular}{|c|c|c|c|c|c|c|c|}
\hline & \multicolumn{3}{|c|}{$\%$ Change in anxiety } & & \multicolumn{3}{|c|}{$\%$ Change in anxiety } \\
\hline Step 1 & & & & Step 1 & & & \\
\hline Arm & -0.06 & -0.49 & 0.63 & Arm & -0.05 & -0.45 & 0.66 \\
\hline Age & -0.13 & -1.09 & 0.28 & Age & -0.12 & -1.03 & 0.31 \\
\hline Baseline anxiety & 0.15 & 1.15 & 0.25 & Baseline anxiety & 0.12 & 0.98 & 0.33 \\
\hline L-Amygdala & 0.02 & 0.17 & 0.86 & R-Amygdala & -0.11 & -0.80 & 0.43 \\
\hline Step 2 & & & & Step 2 & & & \\
\hline L-Amygdala $\times$ Arm & 0.90 & 2.53 & 0.01 & R-Amygdala $\times$ Arm & 0.62 & 1.77 & 0.08 \\
\hline Gender & -0.04 & -0.26 & 0.80 & Gender & -0.03 & -0.23 & 0.82 \\
\hline Age & -0.15 & -1.30 & 0.20 & Age & -0.17 & -1.41 & 0.16 \\
\hline Baseline anxiety & 0.13 & 1.03 & 0.31 & Baseline anxiety & 0.13 & 1.07 & 0.29 \\
\hline L-ventromedial PFC & -0.18 & -1.41 & 0.16 & R-ventromedial PFC & -0.20 & -1.31 & 0.19 \\
\hline Step 2 & & & & Step 2 & & & \\
\hline L-ventromedial PFC $\times$ Arm & 0.28 & 0.70 & 0.48 & R-ventromedial PFC $\times$ Arm & 0.08 & 0.21 & 0.83 \\
\hline Step 1 & & & & Step 1 & & & \\
\hline Arm & -0.07 & -0.58 & 0.56 & Arm & -0.06 & -0.51 & 0.61 \\
\hline ICV & 0.11 & 0.75 & 0.45 & ICV & 0.11 & 0.73 & 0.47 \\
\hline Gender & -0.02 & -0.14 & 0.89 & Gender & -0.06 & -0.39 & 0.70 \\
\hline
\end{tabular}
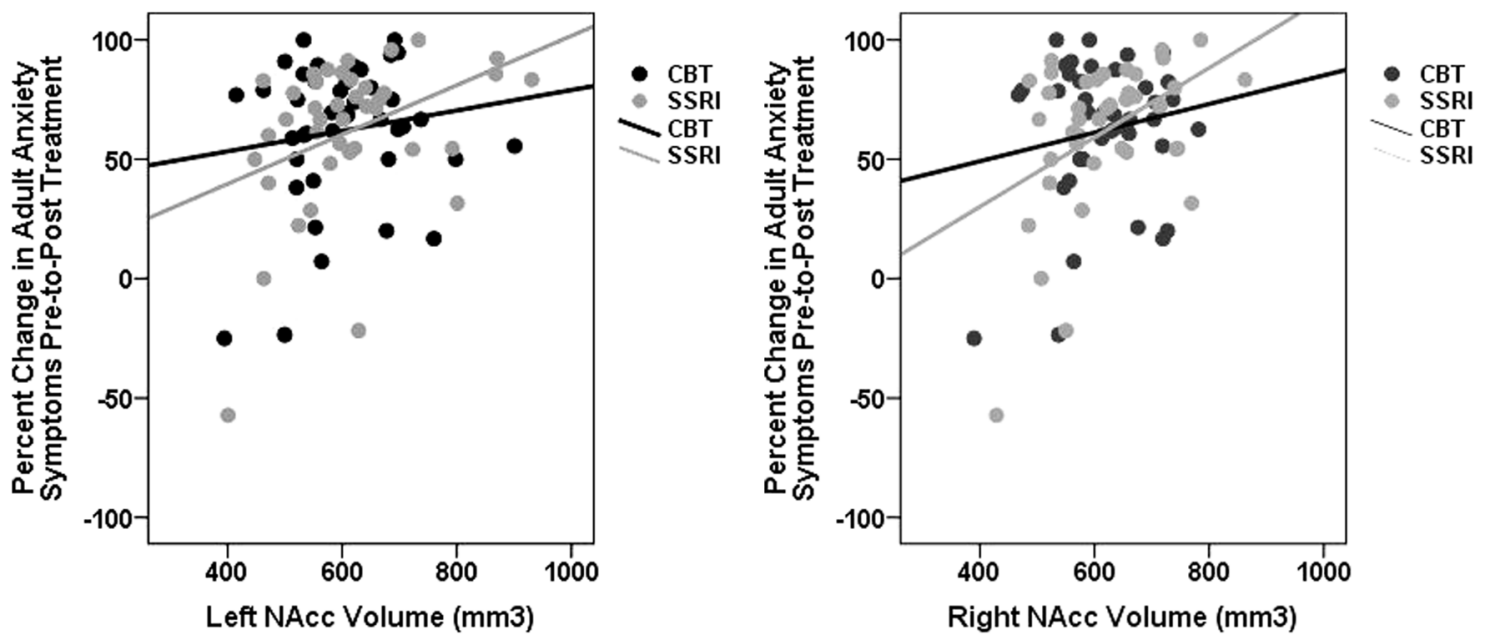

Fig. 1 NAcc volume and adult anxiety treatment response. Scatter plots reflecting the association between baseline left and right NAcc (nucleus accumbens) volume (non-centered) and change in clinician-rated adult anxiety symptoms (HAM-A) following CBT and SSRI treatment (separate colors). CBT cognitive-behavioral therapy, SSRI selective serotonin reuptake inhibitor. 
Table 3. Hierarchical linear regression analyses examining whether baseline ROI volume predicts change in anxiety symptoms pre-to-post treatment among youth (Study 2).

\begin{tabular}{|c|c|c|c|c|c|c|c|}
\hline & \multicolumn{3}{|c|}{$\%$ Change in anxiety } & & \multicolumn{3}{|c|}{$\%$ Change in anxiety } \\
\hline Step 1 & & & & Step 1 & & & \\
\hline Arm & -0.07 & -0.46 & 0.65 & Arm & -0.07 & -0.46 & 0.65 \\
\hline ICV & 0.04 & 0.20 & 0.85 & $\mathrm{ICV}$ & -0.03 & -0.17 & 0.86 \\
\hline Age & 0.01 & 0.04 & 0.97 & Age & -0.01 & -0.03 & 0.97 \\
\hline Baseline anxiety & 0.16 & 1.00 & 0.32 & Baseline anxiety & 0.17 & 1.07 & 0.29 \\
\hline L-Amygdala & -0.07 & -0.38 & 0.71 & R-Amygdala & 0.12 & 0.70 & 0.49 \\
\hline Step 2 & & & & Step 2 & & & \\
\hline L-Amygdala $\times$ Arm & -0.33 & -0.67 & 0.51 & R-Amygdala $\times$ Arm & 0.00 & 0.01 & 0.99 \\
\hline Step 1 & & & & Step 1 & & & \\
\hline Gender & -0.13 & -0.71 & 0.48 & Gender & -0.15 & -0.84 & 0.41 \\
\hline Site & 0.30 & 1.92 & 0.06 & Site & 0.29 & 1.62 & 0.11 \\
\hline Baseline anxiety & 0.21 & 1.31 & 0.20 & Baseline anxiety & 0.17 & 1.05 & 0.30 \\
\hline L-ventromedial PFC & 0.25 & 1.24 & 0.22 & R-ventromedial PFC & -0.01 & -0.04 & 0.97 \\
\hline Step 2 & & & & Step 2 & & & \\
\hline L-ventromedial PFC $\times$ Arm & 0.16 & 0.36 & 0.72 & R-ventromedial PFC $\times$ Arm & 0.27 & 0.56 & 0.58 \\
\hline Step 1 & & & & Step 1 & & & \\
\hline Arm & -0.06 & -0.43 & 0.67 & Arm & -0.10 & -0.66 & 0.51 \\
\hline ICV & -0.12 & -0.64 & 0.52 & ICV & -0.04 & -0.20 & 0.84 \\
\hline Age & 0.14 & 0.89 & 0.38 & Age & 0.08 & 0.49 & 0.63 \\
\hline
\end{tabular}

\section{DISCUSSION}

Structural variations of neural regions implicated in fear and avoidance responses are well implicated in anxiety disorders across youth and adult samples [10]. The current study examined whether gray matter volume of three neural regions supporting fear and avoidance responses (i.e., bilateral amygdala, NACC, and ventromedial PFC) predicted CBT and SSRI treatment outcome in two independent samples of patients with anxiety disorders. Several findings emerged from the current study. First, among the adult sample, greater pre-treatment bilateral NAcc volume was associated with a greater reduction in clinician-rated anxiety symptoms pre-to-post treatment. Results were similar across CBT and SSRI treatment, and these findings were maintained when adjusting for patient's age, sex, and total intracranial brain volume. Notably, this finding was reproduced in an independent sample of children and adolescents with anxiety disorders. Specifically, greater left NAcc volume was also predictive of greater decreases in anxiety symptoms pre-to-post CBT and SSRI treatment among youth with current anxiety. Together, these findings provide promising support for the role of NAcc volume as an objective marker of anxiety treatment improvement that spans across development.

As previously highlighted, the NAcc is involved in modulating behavioral responses to both rewarding and aversive events [71], and there is consistent evidence from animal studies showing that the NAcc plays a key role in both passive and active avoidance behavior [72-74]. In prior studies, GAD and greater anxiety severity is associated with larger NAcc volume among adults [29, 30], and it has been suggested that alterations in NAcC volume may be responsible for avoidancerelated behaviors typically observed among individuals with anxiety [31]. Interestingly, greater baseline NAcc volume was associated with greater treatment gains across two independent samples in the current study. Thus, one interpretation of the current findings is that individuals who exhibit greater preexisting abnormalities in NAcc function may respond more favorably to CBT and SSRI treatment. Indeed, CBT for anxiety works to alter avoidance responses, particularly in response to 


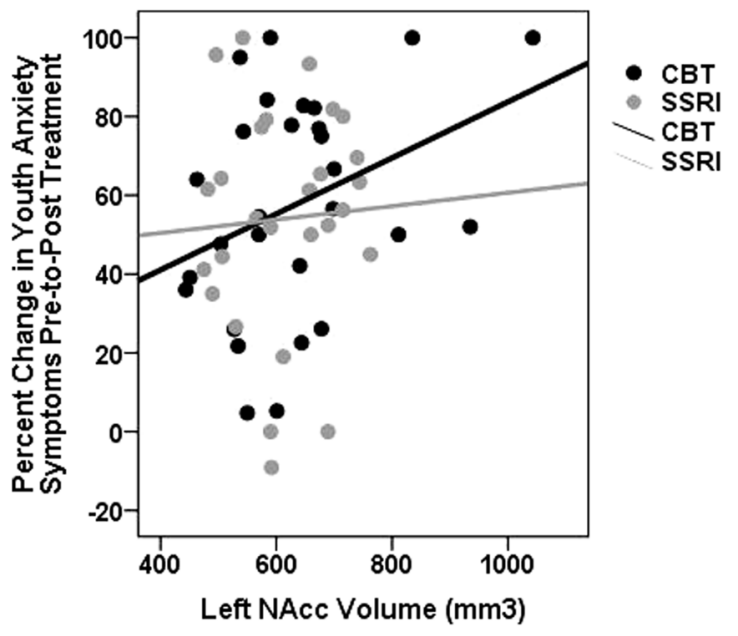

Fig. 2 NAcc volume and youth anxiety treatment response. Scatter plot reflecting the association between baseline left NAcc (nucleus accumbens) volume (non-centered) and change in clinicianrated youth anxiety symptoms (PARS) following CBT and SSRI treatment (separate colors). CBT cognitive-behavioral therapy, SSRI selective serotonin reuptake inhibitor.

threat-relevant material, through exposure therapy. Moreover, there is research showing that SSRI treatment increases extracellular serotonin and dopamine levels in the NAcc [75]. Thus, one interpretation of the current findings is that altering avoidance responses may be a mechanism of action in CBT and SSRI treatment, and that these effects may be most meaningful for individuals who exhibit greater pretreatment deficits in neural systems underlying avoidance behaviors. To fully test this hypothesis, future studies should examine whether CBT or SSRI treatment alters NAcc volume, and if the degree of change corresponds to gradation of anxiety symptom improvement preto-post treatment.

Despite evidence for the role of amygdala and ventromedial PFC in the pathophysiology of anxiety and fear responses [14, 15, 76], the current study found limited evidence for these regions serving as treatment predictors across the two samples. This contrasts with previous functional MRI studies of anxious youth and adults showing that activation of the amygdala and prefrontal regions during the processing of threat-relevant material is associated with anxiety improvement pre-to-post treatment [40, 43-47]. Although prior studies have shown that degree of amygdala volume is associated with amygdala functional activity during emotion processing tasks among patient samples [25], the current findings suggest that amygdala volume may be less implicated in treatment prediction for anxiety disorders, relative to amygdala activation during threat-processing tasks.

Contrary to our expectation, baseline anxiety severity was generally unrelated to gray matter volume of regions implicated in fear and avoidance processes. Consistent with prior studies [19-24], greater baseline anxiety severity was associated with reduced left amygdala volume in the adult sample; however, this was not evident in the child and adolescent sample. Notably, most studies exploring structural gray matter abnormalities in anxiety disorders have focused on group differences (e.g., GAD vs. healthy controls) vs. exploring relations with symptom severity among patients. Moreover, the current study included samples of patients with heterogeneous anxiety disorders and high comorbidity rates, which may not be generalizable to these previous studies. Thus, future studies should continue to explore whether amygdala or ventromedial PFC volume may be more predictive of treatment outcome for patients with certain anxiety diagnoses (e.g., social phobia) vs. comorbid presentations.
Importantly, the present study benefited from the ability to reproduce the NAcc treatment prediction finding in a distinct sample of youth with anxiety disorders. Given that anxiety disorders are most likely to onset during childhood and adolescence [50], identifying biological markers of intervention response for this developmental population is essential. To our knowledge, this is the first study to include a separate independent sample when testing neural predictors of treatment response. The ability to reproduce findings in separate samples is critical for advancing the field of precision medicine. Interestingly, although the two samples were similar in respect to frequency of current anxiety diagnoses (approximately 70\% GAD, 50\% SAD, etc.), there were differences across the two studies that attest to the generalizability of the current findings for anxiety treatment prediction. For instance, the youth study was a multisite, nonrandomized control study and included a lower number of patients with comorbid depression, relative to the adult sample. Patients in the adult study also had more options regarding SSRI medications (vs. sertraline only in the youth study) as a result of more medications being FDA approved in the adult sample and because the adult study was designed to be consistent with the National Institute of Mental Health Research Domain Criteria (RDoC) Initiative and therefore included patients with broad internalizing psychopathologies. Despite developmental and study-specific differences, NAcc volume appears to be a robust predictor of anxiety symptom improvement pre-to-post treatment across youth and adult patients.

There were limitations to the current study which provide important directions for future research. First, the present study focused solely on structural MRI predictors of treatment response. Future treatment outcome studies would benefit from the inclusion of threat avoidance tasks to fully understand the functional role of NAcc in treatment response for anxiety. Second, we chose to utilize an a priori ROI approach focusing on regions implicated in anxiety, fear, and avoidance models to reduce the number of statistical analyses conducted. However, the current study included six predictor regression models for each study; although we were able to reproduce the current findings in a separate independent sample, further replication is warranted. Similarly, there may be other structural MRI predictors of treatment response for anxiety across development that should be explored in future studies. Next, although the focus on patients with heterogeneous anxiety disorders was intentional to increase the generalizability of the current findings to the community and to understand predictors of treatment response for this population, we were unable to examine whether specific anxiety diagnoses moderated any of the above findings because of the high comorbidity rates. Thus, future studies should explore whether findings are specific to comorbid anxiety profiles or are observed for specific diagnoses. Finally, despite NAcc volume doubling the amount of variance accounted for in treatment outcome across both studies, the total proportion of variance in treatment outcome explained by the predictor variables was still relatively low (approximately 20\%), which is not uncommon for treatment outcome studies. Thus, continued work in this area is needed to improve prediction models. For example, combining structural data (e.g., NAcc volume) with other measures of threat processing and avoidance behaviors (e.g., behavioral data, functional imaging) may result in improved accuracy and prediction in future anxiety treatment outcome studies.

In summary, these findings highlight NAcc volume as a predictor of anxiety symptom improvement following CBT and SSRI treatment across two independent samples of patients with anxiety disorders. Future studies are needed to clarify the mechanisms through which NAcc volume exerts its therapeutic effects. However, results from the current study highlight the utility of structural MRI as a tool for predicting which individuals are most likely to benefit from anxiety treatment across development. 


\section{FUNDING AND DISCLOSURES}

This work was supported by National Institute of Mental Health Grant R01-MH086517 to C.S.M. and K.L.P., National Institute of Mental Health grant R01MH101497 (to K.L.P.), and Center for Clinical and Translational Science (CCTS) UL1RR029879. K.L.B. is supported by National Institute of Mental Health grant [MH113793]. The authors declare no competing interests.

\section{ACKNOWLEDGEMENTS}

We would like to thank James Swain, M.D., Ph.D., Gregory Hanna, M.D., Elizabeth Koschmann, Ph.D., David Simpson, Ph.D., L.C.S.W., Sucheta Connolly, M.D., and Jennifer Francis, Ph.D. for their help in providing therapeutic services for the current study.

\section{ADDITIONAL INFORMATION}

Supplementary information is available for this paper at (https://doi.org/10.1038/ s41386-019-0575-5).

Publisher's note Springer Nature remains neutral with regard to jurisdictional claims in published maps and institutional affiliations.

\section{REFERENCES}

1. Baxter AJ, Vos T, Scott KM, Ferrari AJ, Whiteford HA. The global burden of anxiety disorders in 2010. Psychol Med. 2014;44:2363-74.

2. Steel Z, Marnane C, Iranpour C, Chey T, Jackson JW, Patel V, et al. The global prevalence of common mental disorders: a systematic review and meta-analysis 1980-2013. Int J Epidemiol. 2014;43:476-93.

3. Butler AC, Chapman JE, Forman EM, Beck AT. The empirical status of cognitivebehavioral therapy: a review of meta-analyses. Clin Psychol Rev. 2006;26:17-31.

4. Ravindran LN, Stein MB. The pharmacologic treatment of anxiety disorders: a review of progress. J Clin Psychiatry. 2010;71:839-54.

5. Hofmann SG, Asnaani A, Vonk IJ, Sawyer AT, Fang A. The efficacy of cognitive behavioral therapy: a review of meta-analyses. Cogn Ther Res. 2012;36:427-40.

6. Baldwin DS, Anderson IM, Nutt DJ, Allgulander C, Bandelow B, den Boer JA, et al. Evidence-based pharmacological treatment of anxiety disorders, post-traumatic stress disorder and obsessive-compulsive disorder: a revision of the 2005 guidelines from the British Association for Psychopharmacology. J Psychopharmacol. 2014;28:403-39.

7. Bauer I, Wilansky-Traynor P, Rector NA. Cognitive-behavioral therapy for anxiety disorders with comorbid depression: a review. Int J Cogn Ther. 2012;5:118-56.

8. Mobach L, Gould K, Hudson JL Comorbidity in Childhood Anxiety Disorders. In Pediatric Anxiety Disorders 2019 Jan (pp. 277-98). Academic Press.

9. Gadermann AM, Alonso J, Vilagut G, Zaslavsky AM, Kessler RC. Comorbidity and disease burden in the National Comorbidity Survey Replication (NCS-R). Depress Anx. 2012;29:797-806

10. Shin LM, Liberzon I. The neurocircuitry of fear, stress, and anxiety disorders. Neuropsychopharmacology. 2010;35:169-91.

11. Levita L, Hoskin R, Champi S. Avoidance of harm and anxiety: a role for the nucleus accumbens. Neuroimage. 2012;62:189-98.

12. Maren S. Pavlovian fear conditioning as a behavioral assay for hippocampus and amygdala function: cautions and caveats. Eur J Neurosci. 2008;28:1661-6.

13. Quirk GJ, Russo GK, Barron JL, Lebron K. The role of ventromedial prefrontal cortex in the recovery of extinguished fear. J Neurosci. 2000;20:6225-31.

14. Phelps EA, LeDoux JE. Contributions of the amygdala to emotion processing: from animal models to human behavior. Neuron. 2005:48:175-87.

15. Phelps EA, Delgado MR, Nearing KI, LeDoux JE. Extinction learning in humans: role of the amygdala and ventromedial PFC. Neuron. 2004;43:897-905.

16. Delgado MR, Nearing KI, LeDoux JE, Phelps EA. Neural circuitry underlying the regulation of conditioned fear and its relation to extinction. Neuron. 2008;59:829-38.

17. Wendler E, Gaspar JC, Ferreira TL, Barbiero JK, Andreatini R, Vital MA, et al. The roles of the nucleus accumbens core, dorsomedial striatum, and dorsolateral striatum in learning: performance and extinction of Pavlovian fear-conditioned responses and instrumental avoidance responses. Neurobiol Learn Mem. 2014;109:27-36.

18. Kalin NH, Shelton SE, Fox AS, Oakes TR, Davidson RJ. Brain regions associated with the expression and contextual regulation of anxiety in primates. Biol Psychiatry. 2005;58:796-804.

19. Fisler MS, Federspiel A, Horn H, Dierks T, Schmitt W, Wiest R, et al. Spider phobia is associated with decreased left amygdala volume: a cross-sectional study. BMC Psychiatry. 2013;13:70.
20. Hayano F, Nakamura M, Asami T, Uehara K, Yoshida T, Roppongi T, et al. Smaller amygdala is associated with anxiety in patients with panic disorder. Psychiatry Clin Neurosci. 2009;63:266-76.

21. Irle $E$, Ruhleder $M$, Lange $C$, Seidler-Brandler U, Salzer $S$, Dechent $P$, et al. Reduced amygdalar and hippocampal size in adults with generalized social phobia. J Psychiatry Neurosci. 2010;35:126-31.

22. Milham MP, Nugent AC, Drevets WC, Dickstein DS, Leibenluft E, Ernst $M$, et al. Selective reduction in amygdala volume in pediatric anxiety disorders: a voxelbased morphometry investigation. Biol Psychiatry. 2005;57:961-6.

23. Morey RA, Gold AL, LaBar KS, Beall SK, Brown VM, Haswell CC, et al. Amygdala volume changes in posttraumatic stress disorder in a large case-controlled veterans group. Arch Gen Psychiatry. 2012;69:1169-78.

24. Alemany S, Mas A, Goldberg X, Falcón C, Fatjó-Vilas M, Arias B, et al. Regional gray matter reductions are associated with genetic liability for anxiety and depression: an MRI twin study. J Affect Disord. 2013;149:175-81.

25. Siegle GJ, Konecky RO, Thase ME, Carter CS. Relationships between amygdala volume and activity during emotional information processing tasks in depressed and never-depressed individuals: an fMRI investigation. Ann N Y Acad Sci. 2003;985:481-4.

26. De Bellis MD, Casey BJ, Dahl RE, Birmaher B, Williamson DE, Thomas KM, et al. A pilot study of amygdala volumes in pediatric generalized anxiety disorder. Biol Psychiatry. 2000:48:51-57.

27. Schienle A, Ebner F, Schäfer A. Localized gray matter volume abnormalities in generalized anxiety disorder. Eur Arch Psychiatry Clin Neurosci. 2011;261:303-7.

28. Liao W, Xu Q, Mantini D, Ding J, Machado-de-Sousa JP, Hallak JE, et al. Altered gray matter morphometry and resting-state functional and structural connectivity in social anxiety disorder. Brain Res. 2011;1388:167-77.

29. Günther V, Ihme K, Kersting A, Hoffmann KT, Lobsien D, Suslow T. Volumetric associations between amygdala, nucleus accumbens, and socially anxious tendencies in healthy women. Neurosci. 2018;374:25-32.

30. Kühn S, Schubert F, Gallinat J. Structural correlates of trait anxiety: reduced thickness in medial orbitofrontal cortex accompanied by volume increase in nucleus accumbens. J Affect Disord. 2011;134:315-9.

31. Lago T, Davis A, Grillon C, Ernst M. Striatum on the anxiety map: small detours into adolescence. Brain Res. 2017:1654:177-84.

32. Ball TM, Stein MB, Ramsawh HJ, Campbell-Sills L, Paulus MP. Single-subject anxiety treatment outcome prediction using functional neuroimaging. Neuropsychopharmacology. 2014;39:1254-61.

33. Gabrieli JD, Ghosh SS, Whitfield-Gabrieli S. Prediction as a humanitarian and pragmatic contribution from human cognitive neuroscience. Neuron. 2015;85:11-26.

34. Bryant RA, Felmingham K, Whitford TJ, Kemp A, Hughes G, Peduto A, et al. Rostral anterior cingulate volume predicts treatment response to cognitive-behavioural therapy for posttraumatic stress disorder. J Psychiatry Neurosci. 2008;33:142-6.

35. Levy-Gigi E, Szabó C, Kelemen O, Kéri S. Association among clinical response, hippocampal volume, and FKBP5 gene expression in individuals with posttraumatic stress disorder receiving cognitive behavioral therapy. Bio Psychiatry. 2013;74:793-800.

36. Reinecke A, Thilo K, Filippini N, Croft A, Harmer CJ. Predicting rapid response to cognitive-behavioural treatment for panic disorder: the role of hippocampus, insula, and dorsolateral prefrontal cortex. Behav Res Ther. 2014;62:120-8.

37. Bauer EP. Serotonin in fear conditioning processes. Behav Brain Res. 2015;277:68-77.

38. Brooks SJ, Stein DJ. A systematic review of the neural bases of psychotherapy for anxiety and related disorders. Dialogues Clin Neurosci. 2015;17:261-79.

39. Faria V, Appel L, Åhs F, Linnman C, Pissiota A, Frans Ö, et al. Amygdala subregions tied to SSRI and placebo response in patients with social anxiety disorder. Neuropsychopharmacology. 2012;37:2222-32.

40. Gorka SM, Young CB, Klumpp H, Kennedy AE, Francis J, Ajilore O, et al. Emotionbased brain mechanisms and predictors for SSRI and CBT treatment of anxiety and depression: a randomized trial. Neuropsychopharmacology. 2019;44: 1639-48.

41. Phan KL, Coccaro EF, Angstadt M, Kreger KJ, Mayberg HS, Liberzon I, et al. Corticolimbic brain reactivity to social signals of threat before and after sertraline treatment in generalized social phobia. Biol Psychiatry. 2013;73:329-36.

42. Young KS, Burklund LJ, Torre JB, Saxbe D, Lieberman MD, Craske MG. Treatment for social anxiety disorder alters functional connectivity in emotion regulation neural circuitry. Psychiatry Res. 2017;261:44-51.

43. Bryant RA, Felmingham K, Kemp A, Das P, Hughes G, Peduto A, et al. Amygdala and ventral anterior cingulate activation predicts treatment response to cognitive behaviour therapy for post-traumatic stress disorder. Psychol Med. 2008;38:555-61.

44. McClure EB, Adler A, Monk CS, Cameron J, Smith S, Nelson EE, et al. fMRI predictors of treatment outcome in pediatric anxiety disorders. Psychopharmacology. 2007;191:97-105. 
45. Whalen PJ, Johnstone T, Somerville LH, Nitschke JB, Polis S, Alexander AL, et al. A functional magnetic resonance imaging predictor of treatment response to venlafaxine in generalized anxiety disorder. Biol Psychiatry. 2008;63:858-63.

46. Klumpp H, Fitzgerald DA, Angstadt M, Post D, Phan KL. Neural response during attentional control and emotion processing predicts improvement after cognitive behavioral therapy in generalized social anxiety disorder. Psychol Med. 2014;44:3109-21.

47. Kujawa A, Swain JE, Hanna GL, Koschmann E, Simpson D, Connolly S, et al. Prefrontal reactivity to social signals of threat as a predictor of treatment response in anxious youth. Neuropsychopharmacology. 2016;41:1983.

48. Wonderlick JS, Ziegler DA, Hosseini-Varnamkhasti P, Locascio JJ, Bakkour A, Van Der Kouwe A, et al. Reliability of MRI-derived cortical and subcortical morphometric measures: effects of pulse sequence, voxel geometry, and parallel imaging. Neuroimage. 2009;44:1324-33.

49. Elliott ML, Knodt AR, Ireland D, Morris ML, Poulton R, Ramrakha S, et al. Poor testretest reliability of task-fMRI: new empirical evidence and a meta-analysis. bioRxiv. 2019; 681700.

50. Jones PB. Adult mental health disorders and their age at onset. Br J Psychiatry. 2013;202:s5-s10.

51. Boekel W, Wagenmakers EJ, Belay L, Verhagen J, Brown S, Forstmann BU. A purely confirmatory replication study of structural brain-behavior correlations. Cortex. 2015;66:115-33.

52. Button KS, loannidis JP, Mokrysz C, Nosek BA, Flint J, Robinson ES, et al. Power failure: why small sample size undermines the reliability of neuroscience. Nat Rev Neurosci. 2013;14:365.

53. Lovibond SH, Lovibond PF. Manual for the depression anxiety stress scales. Psychology Foundation of Australia; 1996.

54. First MB, Williams JB, Karg RS, Spitzer RL Structured clinical interview for DSM-5Research version (SCID-5 for DSM-5, research version; SCID-5-RV). Arlington, VA: American Psychiatric Association; 2015:1-94.

55. Hamilton MAX. The assessment of anxiety states by rating. B J Med Psychol. 1959;32:50-55.

56. Hamilton MA. A rating scale for depression. J Neurol Neurosurg Psychiatry. 1960;23:56.

57. Barlow DH, Craske MG Mastery of your anxiety and panic. Oxford University Press; 2006.

58. Beck AT (Ed.). Cognitive therapy of depression. Guilford Press; 1979.

59. Craske MG, Barlow DH, O'Leary TA. Mastery of your anxiety and worry. Graywind Publications; 1991.

60. Kaufman J, Birmaher B, Brent D, Rao UMA, Flynn C, Moreci P, et al. Schedule for affective disorders and schizophrenia for school-age children-present and lifetime version (K-SADS-PL): initial reliability and validity data. J Am Acad Child Adolesc Psychiatry. 1997;36:980-8.

61. Research Units of Pediatric Psychopharmacology Anxiety Study Group. The Pediatric Anxiety Rating Scale (PARS): development and psychometric properties. J Am Acad Child Adolesc Psychiatry. 2002;41:1061-9.

62. Kovacs M. Children's depression inventory: Manual. North Tonawanda, NY: MultiHealth Systems; 1992.

63. Kendall PC, Choudhury S, Hudson J, Webb A. The C.A.T. Project Workbook. Ardmore, PA, USA: Worbook Publishing; 2002.

64. Kendall $P C$, Hedtke $K$ Coping. Cat Workbook, 2nd edn. Worbook Publishing: Ardmore, PA, USA; 2006.

65. Dale A, Fischl B, Sereno M. Cortical surface-based analysis: I. segmentation and surface reconstruction. Neuroimage. 1999;9:179-94.

66. Fischl B, Salat D, Busa E, Albert M, Dieterich M, Haselgrove C, et al. Whole brain segmentation: automated labeling of neuroanatomical structures in the human brain. Neuron. 2002;33:341-55.

67. Desikan R, Ségonne F, Fischl B, Quinn B, Dickerson B, Blacker D, et al. An automated labeling system for subdividing the human cerebral cortex on MRI scans into gyral based regions of interest. Neuroimage. 2006;31:968-80.

68. Fischl B. Automatically parcellating the human cerebral cortex. Cereb Cortex. 2004; 14:11-22.

69. Fischl B, Dale A. Measuring the thickness of the human cerebral cortex from magnetic resonance images. Proc Natl Acad Sci. 2000;97:11050-5.

70. Han X, Jovicich J, Salat D, van der Kouwe A, Quinn B, Czanner S, et al. Reliability of MRI-derived measurements of human cerebral cortical thickness: the effects of field strength, scanner upgrade and manufacturer. Neuroimage. 2006;32:180-94

71. Levita L, Hare TA, Voss HU, Glover G, Ballon DJ, Casey BJ. The bivalent side of the nucleus accumbens. Neuroimage. 2009;44:1178-87.

72. Burhans LB, Gabriel M. Contextual modulation of conditioned responses: role of the ventral subiculum and nucleus accumbens. Behav Neurosci. 2007;121:1243-57.

73. Manago F, Castellano C, Oliverio A, Mele A, De Leonibus E. Role of dopamine receptors subtypes, D1-like and D2-like, within the nucleus accumbens subregions, core and shell, on memory consolidation in the one-trial inhibitory avoidance task. Learn Mem. 2009;16:46-52.

74. Schoenbaum G, Setlow B. Lesions of nucleus accumbens disrupt learning about aversive outcomes. J Neurosci. 2003;23:9833-41.

75. Kitaichi $Y$, Inoue $T$, Nakagawa $S$, Boku S, Kakuta A, Izumi $T$, et al. Sertraline increases extracellular levels not only of serotonin, but also of dopamine in the nucleus accumbens and striatum of rats. Eur J Pharm. 2010;647:90-96.

76. Pine DS. Research review: a neuroscience framework for pediatric anxiety disorders. J Child Psychol Psychiatry. 2007;48:631-48. 